DOI: $10.17957 / \mathrm{IJAB} / 15.1783$

http://www.fspublishers.org

Full Length Article

\title{
FaTT10, a Laccasel-Like Polyphenol Oxidase Involved in the Accumulation of Proanthocyanins Monomer in Strawberry Fruit
}

\author{
Guoyan Hou ${ }^{\dagger}$, Min Yang ${ }^{\dagger}$ Yu Long, Binhua Li, Liangxin Wang, Xiaoyang Liu and Ya Luo* \\ College of Horticulture, Sichuan Agricultural University, Chengdu 611130, P. R. China \\ *For correspondence: luoya945@163.com \\ Contributed equally to this work and are co-first authors \\ Received 09 September 2020; Accepted 08 February 2021; Published 10 May 2021
}

\begin{abstract}
Procyanidins (PAs) is the important flavonoids components in strawberry young fruits, which is synthesized through direct polymerization of flavanone-3-ol units. Numerous studies have been done on the synthesis of PAs, while the oxidation/polymerization processes have not been fully explored. AtTT10, a laccasel-like polyphenol oxidase, was recently reported to be participated in the oxidative polymerization of proanthocyanidins. Whether FaTT10 has a positive effect on oxidative polymerization of strawberry fruit PAs is unclear. In this study, FaTT10 in strawberries were isolated and analyzed. Transient overexpression of FaTT10 decreased the accumulation of PAs monomer in strawberry fruit, whereas RNA interference increased the level of anthocyanin and PAs monomer. 4-Dimethylaminocinamaldehyde (DMACA) staining results also showed that lighter blue color in the FaTT10-RNAi fruit and concentrated blue color in the FaTT10overexpression fruit. Meanwhile, PAs monomer showed a drop-and-rise tendency during strawberry fruit development and ripening. Furthermore, the expression of several flavonoids metabolism-related genes were affected by the altered FaTT10 gene expression levels, such as L-phenylalanine ammonia-lyase (PAL); dihydroflavonol reductase (DFR); chalcone isomerase $(\mathrm{CHI})$; flavanone 3-hydroxylase $(\mathrm{F3H})$; 4-coumarate:CoA ligase $(4 \mathrm{CL})$; Cinnamate 4-hydroxylase $(\mathrm{C} 4 \mathrm{H})$; chalcone synthase $(C H S)$; anthocyanin reductase $(A N R)$; lecoanthocyanidins reductase $(L A R)$ and anthocyanin synthase $(A N S)$. In conclusion, these results revealed that FaTT10 is specifically involved in oxidative polymerization of PAs by regulating the expression of flavonoids metabolism-related genes in strawberry fruits. (C) 2021 Friends Science Publishers
\end{abstract}

Keywords: FaTT10; Polymerization; Proanthcocyanins; Strawberry; Transient gene expression

\section{Introduction}

Proanthcocyanins (PAs) are a type of flavonoids formed by the polymerization of flavanone-3-ol units with C6-C3-C6 basic framework in plant, which are also known as condensed tannins. Based on the difference of C6-C3-C6 basic framework in polymerization degree, PAs is divided into monomers, oligomers $(n=2 \sim 5)$ and polymers $(n \geq 6)$ (Monagas et al. 2005). The degree of PA polymerization is closely related to fruit quality, such as the PA of tetramer begin to have bitter and astringent taste in fruit. With the increase of polymerization degree, astringency is also aggravated. PAs fulfils numerous functions, including protecting against microbial pathogens, insect pests, larger herbivores (Dixon et al. 2004) and affecting the flavor and storage quality of red wine (Cosme et al. 2009). PAs is also health beneficial in the human diet to lower the risk of chronic diseases.

The PAs biosynthesis pathways and its regulation have been basically clear through the study of model plants, such as Arabidopsis and Alfalfa. Early biosynthetic genes (EBGs) and late biosynthetic genes (LBGs) constitute PAs biosynthesis (Pelletier et al. 1999; Lepiniec et al. 2006). Chalcone synthase (CHS), chalcone isomerase (CHI), flavonol 3-hydroxylase $(\mathrm{FHH})$ and flavonol 3'-hydroxylase $\left(F 3^{\prime} H\right)$ belong to the EBGs, which participate in precursor biosynthesis for the whole flavonoid biosynthetic pathway. In the LBGs, dihydroflavonol-4-reductase (DFR), leucoanthocyanidin reductase (LAR), anthocyanidin synthase (ANS), anthocyanidin reductase (ANR), TT19 (glutathione S-transferase), TT12 (MATE transporter) and AHA10 $\left(\mathrm{H}^{+}\right.$-ATPase $)$are involved in PAs precursor synthesis as well as PAs compartmetalization, transport, oxidation and modification (Appelhagen et al. 2015). Among the LBGs, $A N R$ and $L A R$, PAs meatabolic specific enzyme, could convert leuco anthocyanidins to flavane 3alcohols, such as catechin (C), gallocatechin (GC), epicatechin (EC) and epigallocatechin (EGC). The next polymerization mechanism of PAs is still to be confirmed.

There are two general assumptions: (1) EC and EGC might be catalyzed by the action of flavane-3-alcohol gall acyltransferase to form flavan 3-O-gallate; (2) Under the

To cite this paper: Hou G, M Yang, Y Long, B Li, L Wang, X Liu, Y Luo (2021). FaTT10, a Laccasel-like polyphenol oxidase involved in the accumulation of proanthocyanins monomer in strawberry fruit. Intl J Agric Biol 25:1222-1230 
effect of plant laccase-like polyphenol oxidase, such as TT10, EC and EGC is condensed to form polyproanthcocyanins in the vacuoles (Zhao et al. 2010). At present, it is generally accepted that the synthesis of anthocyanin and proanthocyanidins is carried out on the cytoplasmic side of endoplasmic reticulum (ER). However, anthocyanin and PAs are widely distributed in cytosol, vacuole, ER, chloroplast, nucleus, small vesicles, apoplastic space, rhizosphere and pollen surfaces, but most of them are stored in vacuoles. How do these metabolites were transported to vacuoles and stored in vacuoles to polymerization, and even secreted to the surface of pollen grains through vacuole membranes and plasma membranes, and then polymerized has not been studied in depth.

Of all the proteins found that may be involved in the transport/polymerization process, Arabidopsis Transparent Testa 10 (TT10) has attracted the considerable interest from researchers. TT10 encodes a laccasel-like polyphenol oxidase which is identified from Arabidopsis seed coat color mutant (transparent seed coat). This protein was secreted to the extraplastid side under the guidance of the front end signal peptide, and may convert colorless, soluble PAs or PAs monomer derivatives into polyproanthcocyanins. Next, the polyproanthcocyanins combines with cell wall proteins to form insoluble substances deposited in seed coat, thus showing the special color of seeds at maturity (Pourcel et al. 2005). There is very limited research about TT10 in nonmodel plant. Recent research showed that silencing of BnTT10 delayed the pigment accumulation of seed coat at the mature stage, and also increased soluble PAs levels in the seed coat by more than three times as much as that in the control. Interestingly, the insoluble PAs content increased by 1.2-1.9 times and the lignin content decreased significantly (Zhang et al. 2013). It is speculated that BnTT10 is involved in the synthesis of proanthocyanidins and lignin. In addition, transforming DkLACl gene into the TT1O mutant of Arabidopsis thaliana could partially or completely restore its phenotype (Hu et al. 2013), which indicated that $D k L A C 1$ also participated in the oxidative polymerization of proanthocyanidins.

Strawberry (Fragaria x ananassa Duch.) is a good source of flavonoids, such as anthocyanins, flavan-3-ols, flavonols and simple phenols (Pérez et al. 1997; Lin-Wang et al. 2014). An integrated study at biochemical and molecular level have been conducted on different developmental stages (Kosar et al. 2004; Almeida et al. 2007) and various strawberry genotypes (Wang and Lewers 2007; Carbone et al. 2009) However, little is known about the transportation, polymerization and storage process of these compounds in strawberry fruits. Considering the important role of flavonoids in the nutritional quality of strawberry, we aimed at functionally characterizing the actual role of FaTT10 in PAs oxidation/polymerization, in hoping to provide a promising gene candidate for manipulating strawberry flavonoids biosynthesis and PAs polymerization.

\section{Materials and Methods}

\section{Plant material}

Strawberry (Fragaria $\times$ ananassa cv. Benihoppe) plants were grown in plastic greenhouse in Chengdu, China. According to the method of Jia et al. (2013), strawberry fruit development and ripening stages were classified in small green (SG), large green (LG), de-greening stage (DG), white (WT), initial red (IR), partial red (PR) and full red (FR), seven developmental stages. At each stage, ten fruits were collected and snap-frozen in liquid nitrogen, and then were quickly stored at $-80^{\circ} \mathrm{C}$ until processed. Three independent biological replicates were in this experiment.

\section{Phylogenetic analysis}

GDR (https://www.rosaceae.org/) and TAIR (https://www.arabidopsis.org/) were used to download protein sequences. Homologues of TT10 genes were identified by using BioEdit. Phylogenetic relationships of the FaTT10 related proteins were constructed with the neighbor-joining statistical methods and the bootstrap method (1000 replications) using the MEGA 7 program.

\section{RNA extraction and cDNA synthesis}

An RNA extraction kit (TaKaRa) was used for the total RNA extraction. RNA sample integrity and concentration were checked by using an Agilent 2100 bioanalyzer and a NanoDrop ND-1000 spectrophotometer. Before generating the first cDNA, the genomic DNA was eliminated, and then cDNA was synthesized using PrimeScript RT Reagent Kit (Takara, Dalian, China) following the manufacturer's protocol. PCR was performed at $37^{\circ} \mathrm{C}$ for $15 \mathrm{~min}$, followed by $85^{\circ} \mathrm{C}$ for $3 \mathrm{sec}$, and then $12^{\circ} \mathrm{C}$ for $15 \mathrm{~min}$.

\section{Plasmid construction}

A 650 bp cDNAs fragment specifically targeting FaTT10 gene was amplified using appropriate primers (sense: 5'CGGGATCCGTCACCATTCATTGGCACGGAGT-3', antisense $5^{\prime}-$ CGGAATTCCAGCGTAAGCAGAGGAAGCCAT-3') cloned into the BAMH I and EcoR I sites of pTRV2. Then, pTRV1, pTRV2, and pTRV2 derivatives pTRV2-TT10 were separately transformed into Agrobacterium strain GV3101 for RNAi. For overexpression of FaTT10 gene, a 1719 bp cDNAs fragment of FaTT10 was amplified using specific primers (FaTT10: sense 5'TGCTCTAGAATGGCTGGGTTTAACAAAATGGGAG3', and antisense 5 CGGGATCCCTAAGACTTGGAGCAAGGAGGCAT-3') cloned into the $B A M H$ I and $X b a$ I sites of pCAMBIA1301. Then pCAMBIA1301, or pCAMBIA1301 derivatives pCAMBIA1301- TT10 was transformed into 
Agrobacterium strain GV3101 for overexpression. Agrobacterium-mediated virus infiltration into strawberry fruits was performed based on the method of Jia et al. (2011). The Agrobacterium suspension was infiltrated into every DG fruit, and then the injected fruit was placed in a growth chamber at $23^{\circ} \mathrm{C}$ under $16 \mathrm{~h}$ light $/ 8 \mathrm{~h}$ dark and 80 90\% relative humidity for 6 days. During this period, the above injection was repeated twice at the next day and the third day after the first infiltration. Each treatment had ten uniformly sized fruit, and with three replicates.

\section{Quantitative real-time PCR (qRT-PCR) analysis}

The qRT-PCR was used to analyse the expression level of FaTT10 gene and structural genes of PA biosynthesis pathway in the FaTT10-overexpression, FaTT10-RNAi and control fruit. The specific qRT-PCR primers designed for structural genes were following: L-phenylalanine ammonialyase (PAL, sense, 5'- CTCGGTCCACTAATTGAAG-3'; antisense, 5'- AATGCCTTGTTCCTTGAA-3' ); Cinnamate 4-hydroxylase $\quad\left(\mathrm{C} 4 \mathrm{H}, \quad\right.$ sense, $5^{\prime}$ TGCCCTTGGCTTCATGACT-3'; antisense, 5'GCTTGACACTACGGAGAAAGGT-3'); coumarate:CoA ligase (4CL, sense, 5'ACAAGACAATCACGAGTTCA-3'; antisense, 5'AGCAGTAGGTGTGGAGAG-3'); chalcone synthase (CHS sense, 5'- CAAGCCTGAGAAGTTAGAAG-3'; antisense, 5'- AAACAACACACAAGCACTA -3'); chalcone isomerase (CHI, sense, 5'CAAGCCTGAGAAGTTAGAAG-3'; antisense, 5'AAACAACACACAAGCACTA-3'); flavanone 3hydroxylase (F3H, sense, 5'ATCACCGTTCAACCTGTGGAAG-3'; antisense, 5'TCTGGAATGTGGCTATGGACAAC-3'); dihydroflavonol reductase (DFR, sense, 5'- GCTACATCTGTTCATCAC-3'; antisense, $\quad 5$ '-GTCAAGTTCTCCTCAATG-3'); anthocyanin reductase (ANR, sense, 5'CCTGAATACAAAGTCCCGACTGAG-3'; antisense, 5'GTACTTGAAAGTGAACCCCTCCTTC-3'); anthocyanin synthase (ANS, sense, 5'-GCTGCTCTCATCTCAACTAA3'; antisense, 5'- CCTGCTCAACATCGTCTT -3); lecoanthocyanidins reductase ( $L A R$, sense, 5'GGTGATGGCACGGTTAAAGC-3'; antisense, 5'CTCCCACAGTGAAGCAAGTCC -3'); and Actin (sense, 5'-TTCACGAGACCACCTATAACTC-3'; antisense, 5'GCTCATCCTATCAGCGATT -3'). qRT-PCR procedures was: holding for $3 \mathrm{~min}$ at $95^{\circ} \mathrm{C}$, followed by 40 cycles for $10 \mathrm{~s}$ at $95^{\circ} \mathrm{C}$, annealing for $30 \mathrm{~s}$ at $55^{\circ} \mathrm{C}$ and $72^{\circ} \mathrm{C}$ for $15 \mathrm{~s}$. Relative fold changes in gene expression were analyzed using the $2^{-\Delta \Delta \mathrm{CT}}$ method.

\section{4-dimethylaminocinamaldehyde (DMACA) staining assay}

DMACA staining method was used for the observation of PAs accumulation patterns in FaTT10 RNAi and overexpression fruit ( $\mathrm{Li}$ et al. 1996). Longitudinal sections of fruits about $0.2 \mathrm{~cm}$ thick were soaked and decolorized in ethanol solution $(3: 1, \mathrm{v} / \mathrm{v})$ and glacial acetic acid for $12-20$ $\mathrm{h}$, and rinsed with $75 \%$ ethanol for $12 \mathrm{~h}$, then rinsed with distilled water. After dyeing with $0.6 \%$ (w/v) DMACA solution in a cold mixture of methanol and 6 M HCL (1:1, $\mathrm{v} / \mathrm{v}$ ) for $2 \mathrm{~min}$, photographic records were taken.

\section{Determination of PAs monomer and anthocyanin content}

PAs monomer content was determined by using the PAs determination kit (Shagnhai MLBIO Biotechnology Co., Ltd. and Suzhou Keming Biotechnology Co., Ltd.). The quantification of total anthocyanin was measured by the $\mathrm{pH}$ differential spectrophotometry method (Cheng and Breen 1991). Briefly, the extraction was conducted from $0.5 \mathrm{~g}$ mixed strawberry fruit homogenized with $1.8 \mathrm{~mL}$ of cold $1 \%$ $\mathrm{HCl}$-ethanol. Then, the homogenate was centrifuged at 8 , $000 \times g$ for $25 \mathrm{~min}$ at $4^{\circ} \mathrm{C}$. Next, the supernatants were collected for determination of the total anthocyanin content. Anthocyanin concentration was expressed as mg pelargonidin 3-glucoside equivalents $100 \mathrm{~g}^{-1}$ of fresh weight.

\section{Confocal microscopy}

Full coding sequence of the FaTT10 was amplified with primer subc-TT10-F and subc-TT10-R. The open reading frame was then fused to the N-Terminal of the GFP marker in a modified pC1302-35S-GFP vector after Nco I and Spe I digestion. The correct construct was subsequently transformed into the Agrobacterium tumefaciens strain GV3101 and transiently expressed in Nicotiana benthamiana leaves epidermal cell. Stains with the marker (ABRC stock CD3-1003) were used as positive and reference control. GFP signals were captured by Nikon A1 Confocal Laser Microscope.

\section{Results}

Phylogenetic tree of TT10-like homologues for strawberry

To further identify the characteristics of FaTT10, a neighbor-joining phylogenetic tree was constructed with 1000 bootstrap replicates. Scale bars indicate 0.05 estimated amino acid substitutions per site. Numbers above branches indicate the reliability of the associated taxa clustering. The phylogenetic tree showed FaTT10 is more closely related to Fragaria vesca than Pyrus bretschneideri, but clearly distant from that Arabidopsis (Fig. 1).

\section{Changes of PAs monomer content during strawberry fruit development}

PAs monomer content was measured during strawberry fruit development. The results showed that the PAs monomer 
content was mainly accumulating at early and late stage during fruit development. The lowest PA monomer was observed at WT stage, which was $6.56 \mathrm{mg} 100 \mathrm{~g}^{-1} \mathrm{FW}$, and then followed by a gradual increase and reached a highest value at FR stage, which was $71 \mathrm{mg} 100 \mathrm{~g}^{-1} \mathrm{FW}$ and increased by 10.83 fold compared with that of the fruit at WT stage (Fig. 2).

\section{Silencing of the FaTT10 promoting the accumulation of proanthocyanidins monomer}

To test the role of FaTT10 in PAs polymerization, FaTT10 was knocked down by RNAi. Thirty fruits with pedicel were injected with the FaTT10-RNAi vector in vitro. Six days after infiltration, the phenotypic changes of the control and RNAi fruits were observed. The fruit phenotype showed that strawberry fruits grew normally, and there were no adverse symptoms such as fruit shrinkage, mold growth and virus infection in vitro, which indicated that the control and FaTT10-RNAi fruits could be inoculated with Agrobacterium in vitro and did not affect the observation of strawberry fruits phenotype after gene silencing. The results showed that there were more colored fruits in FaTT10RNAi group than that in control (Fig. 3A, B). Meanwhile, qRT-PCR results showed that FaTT10 expression level was significantly down-regulated by 0.54 fold in the FaTT10RNAi fruit compared with the control (Fig. 3C), and the content of anthocaynin and PAs monomer increased by 3.0fold and 2.81fold, respectively (Fig. 3D, E).

PAs are colorless before oxidation, and blue products are produced when they react with DMACA reagents. Therefore, DMACA reagents are commonly used to stain fresh plant tissues. The distribution and content of PAs can be determined by the localizaiton and color of blue products. In this experiment, PAs were located in the control and FaTT10-RNAi fruit tissue and seemed to be more concentrated in the stele of fruit, followed by the pith, the vascular bundle, and the cortex (Fig. 3F). Furthermore, the blue color of the stele, the pith and the vascular bundle in control was more concentrated compared with that of FaTT10-RNAi fruit, which suggested that silencing FaTT10 gene could inhibit the accumulation of PAs in strawberry fruit. Taken together, silencing of FaTT10 gene up-regulated the content of anthocyanin and PAs monomer, which led to the inhibition of PAs content in strawberry fruit.

\section{Overexpression of FaTT10 inhibiting the accumulation of proanthocyanidins monomer}

To further explore FaTT10 gene function in PAs polymerization of strawberry fruit, overexpression FaTT10 via agro-infiltration was also applied in this study. The control and the TT10-overexpression fruit showed the similar fruit phenotype on the sixth day after Agrobacterium injection, which fruit surface was white or partly red (Fig. 4A, B). Meanwhile, qRT-PCR results demonstrated that the

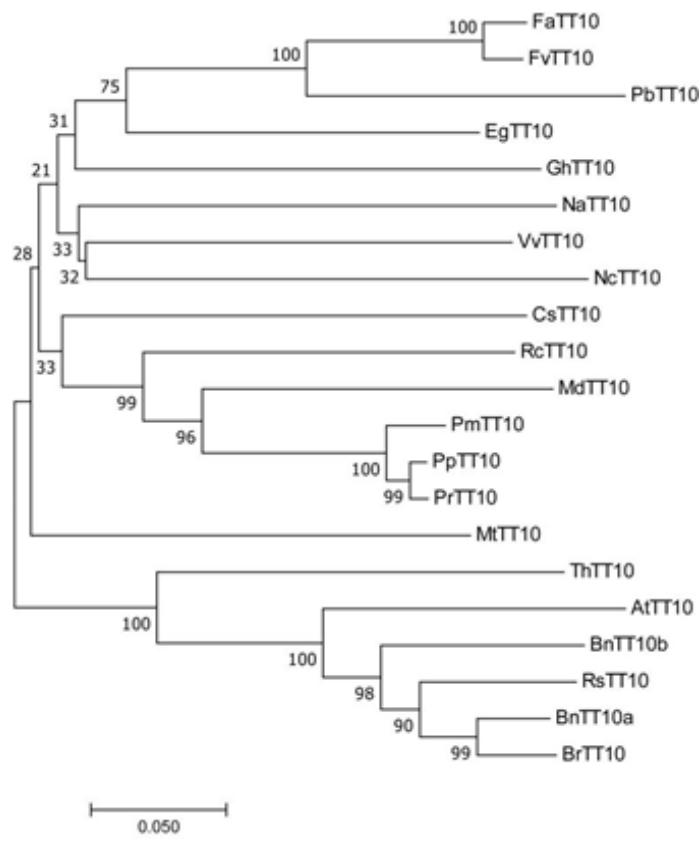

Fig. 1: Phylogenetic analyses of FaTT10 and its closest relatives. Phylogenetic analysis was performed by a neighbor-joining algorithm using MEGA 7 program. Gene IDs were listed as: Prunus persica PpTT10 XP_007200191, Prunus dulcis PrTT10 VVA36043, Prunus mume PmTT10 XP 008237408, Malus domestica MdTT10 XP_028965267, Pyrus bretschneideri PbTT10 XP_009351840, Fragaria vesca FvTT10 XP_011464921, Citrus sinensis CsTT10 XP_006473165, Vitis vinifera VvTT10 XP_002272689, Gossypium hirsutum GhTT10 XP_016748740, Arabidopsis thaliana AtTT10 NC_003076, Brassica napus BnTT10a ADV03954, Brassica napus BnTT10b ADV03952, Brassica rapa BrTT10 XP_009129702, Raphanus sativus RsTT10 XP_018435646.1, Tarenaya hassleriana ThTT10 XP_010521293, Medicago truncatula MtTT10 XP_003603035, Rosa chinensis RcTT10 XP_024189647, Eucalyptus grandis EgTT10 XP_010031937, Nicotiana attenuata NaTT10 XP_019264267, Nymphaea colorata NcTT10 XP_031502939

transcriptional level of FaTT10 was significantly upregulated by 11 fold in the FaTT10-overexpression fruit compared with that of the control fruit (Fig. 4C), which meant that the FaTT10 gene was successfully overexpressed in the strawberry fruits. Furthermore, almost no anthocaynin was detected (data not listed), but PAs monomer decreased by 0.23 fold (Fig. 4D). DMACA dyeing showed that the spatial accumulation of PAs was also in the stele, pith, vascular bundle and cortex of FaTT10-overexpression and control fruit, and concentrated blue color was mainly observed in the stele, and pith of FaTT10-overexpression fruit compared with that of control fruit (Fig. 4E). Above all, overexpression FaTT10 gene inhibited the PAs monomer content, while promoted the accumulation of PAs.

Alteration of FaTT10 genes expression affects the transcripts of PAs biosynthesis-related genes

In order to explore the mechanism of FaTT10 gene in the 


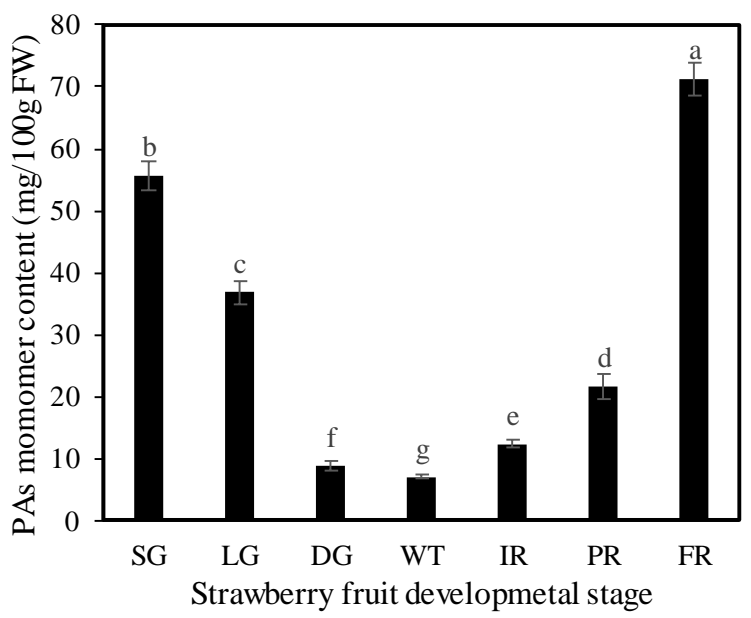

Fig. 2: PAs monomer content during strawberry fruit development. Small green (SG, $7 \mathrm{~d}$ after anthesis), large green (LG, $14 \mathrm{~d}$ after anthesis), de-greening (DG, $18 \mathrm{~d}$ after anthesis), white (WT, $20 \mathrm{~d}$ after anthesis), initial red (IR, $23 \mathrm{~d}$ after anthesis) and full red (FR, $28 \mathrm{~d}$ after anthesis). Values are means \pm SD of three biological replicates. An overall significant difference $(P \leq 0.5)$ is represented by different lower-case letters as determined by Duncan's multiple range test

A

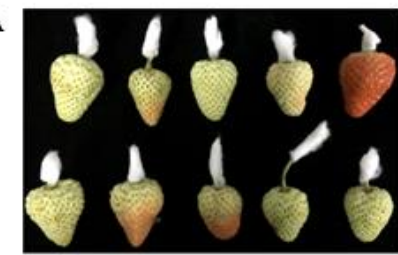

C

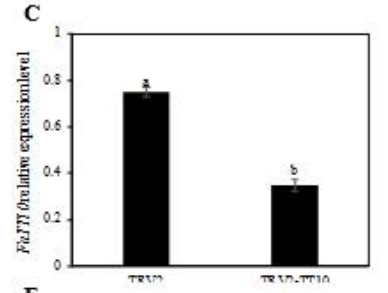

E

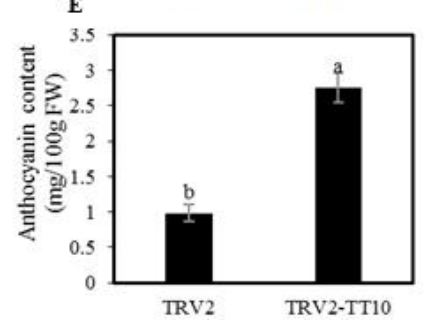

B
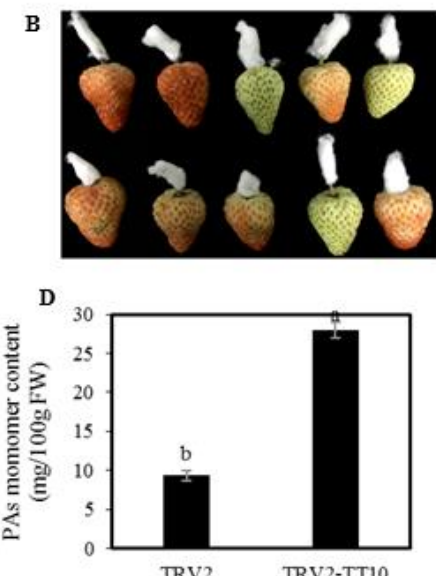

$\mathbf{F}$ TRV2

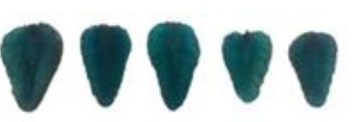

TRV2-TT10

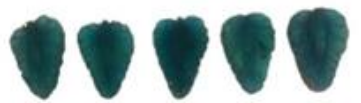

Fig. 3: VIGS for the FaTT10 gene in strawberry fruit. (A) The phenotype of control fruit inoculated with Agrobacterium containing TRV2 in vitro. (B) The phenotype of virus-induced FaTT10 silencing in strawberry fruits. (C) FaTT10 expression level in the control and RNAi fruits by qRT-PCR. (D) PAs monomer content in the control and FaTT10-RNAi fruits. (E) Anthocaynin content in the control and FaTT10-RNAi fruits. (F) DMACA staining of longitudinal sections of the control and FaTT10-RNAi fruits. Values are means \pm SD of three biological replicates. An overall significant difference $(P \leq 0.5)$ is represented by different lower-case letters as determined by Duncan's multiple range test

regulation of strawberry PAs biosynthesis, a set of genes related to flavonoids metabolism, such as L-phenylalanine ammonia-lyase $(P A L)$; cinnamate 4-hydroxylase $(C 4 H)$; 4 coumarate $\mathrm{CoA}$ ligase (4CL); chalcone synthase (CHS); chalcone isomerase $(\mathrm{CHI})$; flavanone 3-hydroxylase $(\mathrm{F} 3 \mathrm{H})$; dihydroflavonol reductase $(D F R)$; anthocyanin reductase
(ANR); anthocyanin synthase (ANS) and lecoanthocyanidins reductase $(L A R)$, were measured using both overexpression and RNAi fruit. The results showed that there were significant differences in the expression levels of the above genes between the RNAi fruit and the control fruit, except ANS gene (Fig. 5A). According to the characteristics of 

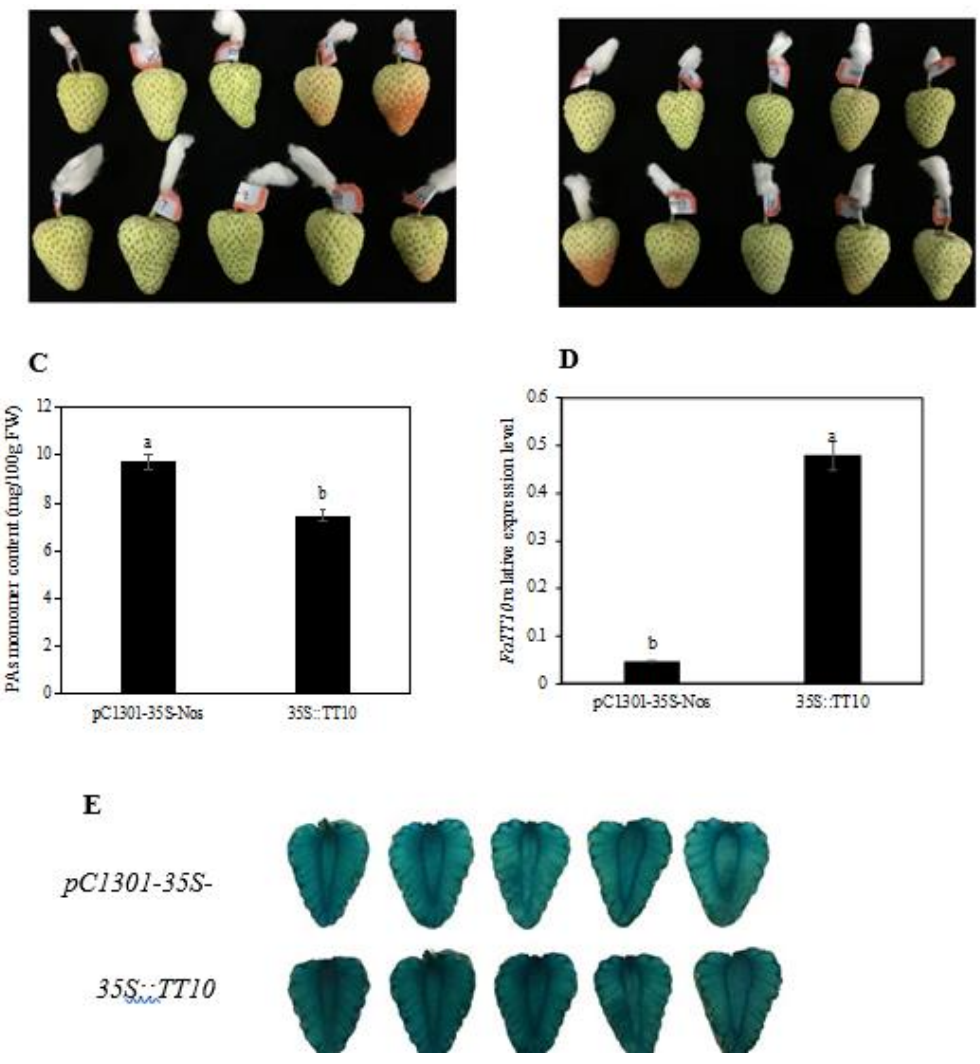

Fig. 4: Overexpression for the TT10 gene in strawberry fruit. (A) The control fruit phenotype on the $6^{\text {th }}$ day in vitro. (B) The FaTT10 overexpression fruit phenotype on the $6^{\text {th }}$ day in vitro. (C) FaTT10 expression level in the control and overexpression fruits. (D) PAs monomer content in the control and overexpression fruits. (E) DMACA staining of longitudinal sections of the control and overexpression fruits. Values are means \pm SD of three biological replicates. An overall significant difference $(\mathrm{P} \leq 0.5)$ is represented by different lower-case letters as determined by Duncan's multiple range test
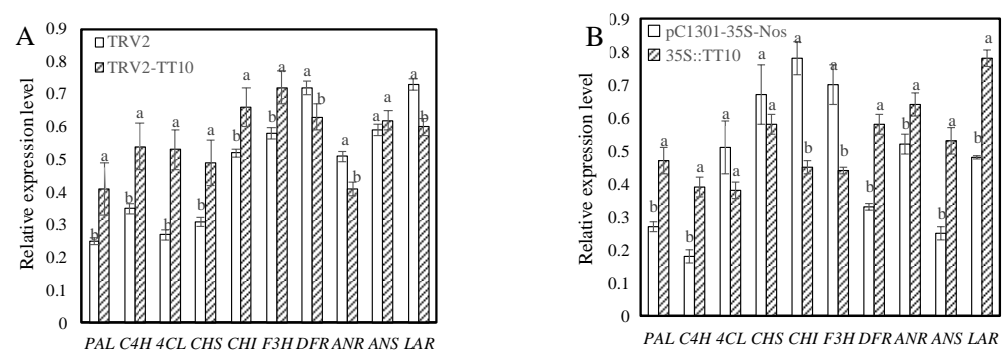

Fig. 5: Alteration of FaTT10 expression affects transcripts of a set of PAs biosynthesis related genes in silencing and overexpressing fruit. Values are means \pm SD of three biological replicates. (A) mRNA expression level of PAs biosynthesis relevant genes in RNAi fruit. (B) mRNA expression level of PAs biosynthesis relevant genes in overexpression fruit

genes expression level, it can be roughly divided into two categories: one is that the expression level in RNAi-FaTT10 fruit was significant up-regulated compared with that in control fruit, including $P A L, C 4 H, 4 C L, C H S, C H I$ and $F 3 H$ (Fig. 5A). The expression levels of $4 C L$ were up-regulated by more than twice, $P A L$ and $C 4 H$ were up-regulated by about $60 \%, \mathrm{CHS}, \mathrm{CHI}$ and $\mathrm{FHH}$ were up-regulated by 48, 29 and $24 \%$, respectively. Another kind of genes, including $D F R, A N R$ and $L A R$, which expression levels were downregulated by 14,20 and $19 \%$ in RNAi-FaTT10 fruit compared with that in control fruit, respectively (Fig. 5A).

In addition, the expression level of PAs biosynthesis related genes was also quantified in FaTT10 overexpression fruit and control fruit. Among these structural gene of PAs biosynthesis, the expression level of $P A L, C 4 H, D F R, A N R$, $A N S$ and $L A R$ genes was significant up-regulated in FaTT10 overexpression fruit compared with that in control fruit, while the expression level of $\mathrm{CHI}$ and $\mathrm{F} 3 \mathrm{H}$ genes was significant down-regulated (Fig. 5B). Taken together, these changes in the expression of genes associated with the 


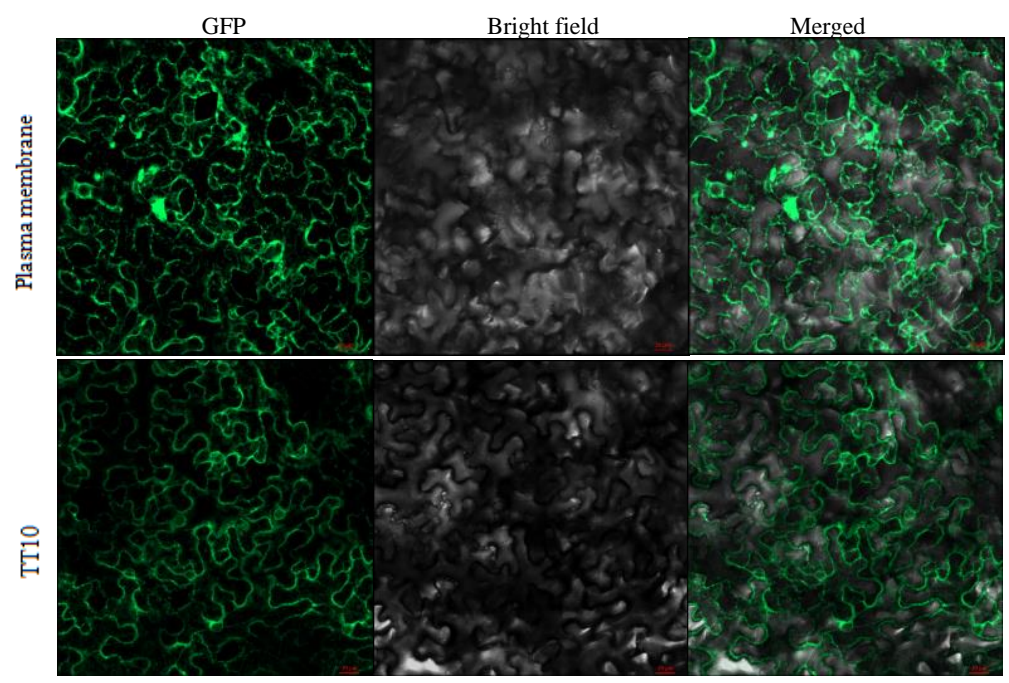

Fig. 6: Sub-celluar localization of strawberry TT10 protein in the epidemis of Nicotiana benthamiana leaves. Left, UV-excited GFP green fluorescence signals. Middle, bright field. Right, merged image. Scale bars $=20 \mu \mathrm{m}$

biosynthesis of PAs in FaTT10 RNAi and overexpression strawberry fruit suggested that FaTT10 were involved in the regulation of PAs biosynthesis by regulating the expression levels of PAs biosynthesis-related genes.

\section{Sub-celluar localization of FaTT10}

After $36 \mathrm{~h}$ infection with Agrobacterium containing pC1302-35S-GFP::TT10 vector and pC1302-35S-GFP empty vector respectively, the epidemis of Nicotiana benthamiana leaves were observed by fluorescence confocal microscope. The results showed that the GFP signal was mainly detected at the plasma membrane in cells transfected with the empty vector and in cells expressing the TT10 fusion proteins (Fig. 6), suggesting that TT10 might be membrane proteins.

\section{Discussion}

Laccase (EC 1.10.3.2) is ubiquitous in bacteria, fungi, insects and higher plants. The research on fungi laccase are the most reported, which function is associated with enhancing virulence of pathogenic bacteria (Salas et al. 1996), lignin degradation (Eggert et al. 1997) and pigment synthesis (Fang et al. 2010). The research on plant laccase is mainly focused on lignin synthesis (Sterjiades et al. 1992; Bao et al. 1993; Ranocha et al. 2002) stress response (Li and Steffens 2002) and pigment synthesis (Cai et al. 2006). Through our previous gene cloning, sequence analysis and subcellular localization prediction, it was showed that the protein encoded by FaTT1O gene belongs to the laccase family and anchors at plasma membrane (Mo et al. 2019). In this study, sub-celluar localization experiment confirmed TT10 located in the plasma membrane in present study. Phylogenetic analysis strengthened TT10 share common genetic ancestry in Rosacea species because of close evolutionary relationships (Fig. 1). In future work, it would be interesting to explore the overall evolutionary history of the whole TT10 family from a wider species range.

Strawberry was identified as potential sources of Btype proanthocyanidins. (Epi) catechin and (epi) afzelechin was always the terminal unit of proanthocyanidin oligomers (Gu et al. 2003). Through proanthocyanidin determination of 15 strawberry cultivars in Spain, it was found that the concentration of proanthocyanidin ranged from $53.9 \mathrm{mg} 100$

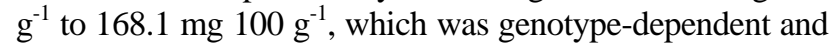
(epi) catechin was the main proanthocyanidin oligomers in strawberry fruit (Buendía et al. 2010). In the previous study, it has been reported that decreased PAs coupled with the decreasing FaTT10 expression level during strawberry fruit development and ripening (Mo et al. 2019), while the content of PAs monomers decreased gradually in fruit from SG to WT stage and then followed by a gradual increased from WT to FR stage (Fig. 2). The simplest hypothesis for this is that FaTT10 specifically regulates the content of PAs monomers could be an integrate part of regulation mechanism for oxidative polymerization of PAs.

In present study, our results showed that inhibition of FaTT10 genes results in more colored fruit (Fig. 3B) and an increase in the level of anthocyanin and PAs monomer (Fig. 3D) in strawberry fruit. In contrast, overexpression of FaTT10 genes can decrease the accumulation of PAs monomer (Fig. 3D). Similar results were obtained in Brassica napus and Arabidopsis, showing that the content of soluble PAs in AtTT10 mutant seeds (Pourcel et al. 2005) and BnTT10-RNAi seeds (Zhang et al. 2013) was higher than that in wild type. In addition, DMACA staining results also showed that lighter blue color in the FaTT10-RNAi fruit (Fig. 3F) and concentrated blue color in the FaTT10overexpression fruit (Fig. 3E) were observed. In Chinese PCNA persimmon, the concentrated blue color was observed in overexpression $D k L A C l$ leaves by DMACA staining, as 
well as the increased soluble and insoluble PA (Mo 2015).

The end products of the flavonoid biosynthetic pathway are PAs. Various MYB-bHLH-WDR (MBW) protein complexes and flavonoid synthesis structure gene (ANR, LAR and ANS) (Feng et al. 2013; Xiao et al. 2014) are involved in the regulation of PAs synthesis at the transcriptional level (Xu et al. 2014a; Xu et al. 2015). In present study, alternation of FaTT10 expression level affected the flavonoid biosynthetic pathway, which includes up-regulation of PAL,C4H,DFR, ANR, ANS and LAR expression level (Fig. 5B) in FaTT10-overexpression fruit, and down-regulation of LBGs (DFR, ANR and LAR) transcriptional level (Fig. 5A) in FaTT10-RNAi fruit compared with respective control. In note, down-regulation of $D F R, A N R$ and $L A R$ was also observed during strawberry fruit development (Xu et al. 2014b).

\section{Conclusion}

In this study, PAs monomer contents first decreased and then increased gradually during strawberry fruit development and ripening. Transient knock-down of FaTT10 or overexpression increased or decreased the level of PAs monomer, accompanied by the changes in PAs contents and gene expression on flavonoids synthesis pathway. The above results indicate that FaTT10 contributes to proanthocyanins polymerization.

\section{Acknowledgements}

This study was supported by the National Natural Science Foundation of China (3180817); the State Education Ministry, Key projects of Sichuan Provincial Education Department (172A0319) and Key projects of Sichuan Provincial Science and Technology Department (2018NZ0126) and Service Station Projects of New Rural Development Research Institute, Sichuan Agricultural University $(2018,2020)$.

\section{Author Contributions}

Ya Luo was responsible for the design of the experiments and the main writing of the manuscript. Most of the experiments and data analysis was completed by Gouyan Hou and Min Yang. All authors participated in the discussion of the results and comments on the manuscript.

\section{Conflict of Interest}

All the authors declare no conflict of interest.

\section{Data Availability}

All data included in this study are available upon request by contact with the corresponding author.

\section{Ethics Approval}

This article does not contain any studies with human participants or animals performed by any of the authors.

\section{References}

Almeida JRM, ED'Amico, A Preuss, F Carbone. CHR Vos, B Deiml, F Mourgues, G Perrotta, TC Fischer, AG Bovy, S Martens, C Rosati (2007). Characterization of major enzymes and genes involved in flavonoid and proanthocyanidin biosynthesis during fruit development in strawberry (Fragaria $\times$ ananassa). Arch Biochem Biophys 465:6171-6181

Appelhagen I, N Nordholt, T Seide, K Spelt, R Koes, F Quattrochio, M Sagasser, B Weisshaar (2015). TRANSPARENT TESTA 13 is a tonoplast $\mathrm{P}_{3 \mathrm{~A}}$-ATPase required for vacuolar deposition of proanthocyanidins in Arabidopsis thaliana seeds. Plant $J$ 82: 840-849

Bao W, DM O'malley, R Whetten, RR Sederoff (1993). A Laccase associated with lignification in Loblolly Pine xylem. Science 260:672-674

Buendía B, MI Gil, JA Tudela, AL Gady, JJ Medina, C Soria, JM López, FA Tomás-Barberán (2010). HPLC-MS Analysis of Proanthocyanidin Oligomers and Other Phenolics in 15 Strawberry Cultivars. J Agric Food Chem 58:3916-3926

Cai XN, EJ Davis, J Ballif, MX Liang, E Bushman, V Haroldsen, J Torabinejad, YJ Wu (2006). Mutant identification and characterization of the laccase gene family in Arabidopsis. J Exp Bot 57:2563-2569

Carbone F, A Preuss, RCH Vos, E_D'Amico, G Perrotta, AG Bovy, S Martens, C Rosati (2009). Developmental, genetic and environmental factors affect the expression of flavonoid genes, enzymes and metabolites in strawberry fruits. Plant Cell Environ 32:1117-1131

Cheng GW, PJ Breen (1991). Activity of phenylalanine ammonia-lyase (PAL) and concentrations of anthocyanins and phenolics in developing strawberry fruit. J Amer Soc Hortic Sci 116:865-869

Cosme F, JM Ricardo-Da-Silva, O Laureano (2009). Effect of various proteins on different molecular weight proanthocyanidin fractions of red wine during wine fining. Amer J Enol Viticult 60:74-82

Dixon RA, DY Xie, SB Sharma (2004). Proanthocyanidins - a final frontier in flavonoid research? New Phytol 165:9-28

Eggert C, U Temp, KEL Eriksson (1997). Laccase is essential for lignin degradation by the white-rot fungus Pycnoporus cinnabarinu. FEBS Lett 407:89-92

Fang WG, EKK Fernandes, DW Roberts, MJ Bidochka, RJS Leger (2010). A laccase exclusively expressed by Metarhizium anisopliae during isotropic growth is involved in pigmentation, tolerance to abiotic stresses and virulence. Fung Genet Biol 47:602-607

Feng HJ, XH Tian, YC Liu, YJ Li, XY Zhang, BJ Jones, YQ Sun, J Sun (2013). Analysis of flavonoids and the flavonoid structural genes in brown fiber of upland cotton. PLoS One 8; Article e58820

Gu LW, MA Kelm, JF Hammerstone, G Beecher, J Holden, D Haytowitz, RL Prior (2003). Screening of foods containing proanthocyanidins and their structural characterization using LC-MS/MS and thiolytic degradation. J Agric Food Chem 51:7513-7521

Hu QN, C Luo, QL Zhang, ZR Luo (2013). Isolation and characterization of a Laccase gene potentially involved in proanthocyanidin polymerization in oriental persimmon (Diospyros kaki Thunb.) fruit. Mol Biol Rep 40:2809-2820

Jia HF, YH Wang, MZ Sun, BB Li, Y Han, YX Zhao, XL Li, N Ding, C Li, WL Ji, WS Jia (2013). Sucrose functions as a signal involved in the regulation of strawberry fruit development and ripening. New Phytol 198:453-465

Jia HF, YM Chai, CL Li, D Lu, JJ Luo, L Qin, YY Shen (2011). Abscisic acid plays an important role in the regulation of strawberry fruit ripening. Plant Physiol 157:188-199

Kosar M, E Kafkas, S Paydas, KHC Baser (2004). Phenolic composition of strawberry genotypes at different maturation stages. J Agric Food Chem 52:1586-1589 
Lepiniec L, I Debeaujon, JM Routaboul, A Baudry, L Pourcel, N Nesi, M Caboche (2006). Genetics and biochemistry of seed flavonoids. Annu Rev Plant Biol 57:405-430

Li L, JC Steffens (2002). Overexpression of polyphenol oxidase in transgenic tomato plants results in enhanced bacterial disease resistance. Planta 215:239-247

Li YG, G Tanner, P Larkin (1996). The DMACA-HCl protocol and the threshold proanthocyanidin content for bloat safety in forage legumes. J Sci Food Agric 70:89-101

Lin-Wang K, TK McGhie, M Wang, Y Liu, B Warren, R Storey, RV Espley, AC Allan (2014). Engineering the anthocyanin regulatory complex of strawberry (Fragaria vesca). Front Plant Sci 5; Article 651

Mo LR (2015). Isolation and Characterization of ADH and PDC Genes Involved in Natural Loss of Astringency in Chinese PCNA Persimmon. Huazhong Agricultural University, Wuhan, Hubei, China

Mo Q, YJ Ling, F Mo, C Ge, S Luo, Y Luo (2019). Cloning and Expression Analysis of FaTT10 in Fragaria ananassa. Genomics Appl Biol 38:699-706

Monagas M, B Hernández-Ledesma, I Garrido, PJ Martín-lvarez, C GómezCordovés, B Bartolomé (2005). Quality assessment of commercia dietary antioxidant products from Vitis vinifera L. grape seeds. Nutr Cancer 53:244-254

Pelletier MK, IE Burbulis, B Winkel-Shirley (1999). Disruption of specific flavonoid genes enhances the accumulation of flavonoid enzymes and end-products in Arabidopsis seedlings. Plant Mol Biol 40:45-54

Pérez AG, R Olías, J Espada, JM Olías, C Sanz (1997). Rapid determination of sugars, nonvolatile acids, and ascorbic acid in strawberry and other fruits. J Agric Food Chem 45:3545-3549

Pourcel L, JM Routaboul, L Kerhoas, M Caboche, L Lepiniec, I Debeaujon (2005). TRANSPARENT TESTA 10 encodes a laccase-like enzyme involved in oxidative polymerization of flavonoids in Arabidopsis seed coat. Plant Cell 17:2966-2980
Ranocha P, M Chabannes, S Chamayou, S Danoun, A Jauneau, AM Boudet, D Goffner (2002). Laccase down-regulation causes alterations in phenolic metabolism and cell wall structure in poplar. Plant Physiol 129:145-155

Salas SD, JE Bennett, KJ Kwon-Chung, JR Perfect, PR Williamson (1996). Effect of the laccase gene, CnLAC1, on virulence of Cryptococcus neoforman. J Exp Med 184:377-386

Sterjiades R, JFD Dean, KEL Eriksson (1992). Laccase from sycamore maple (Acer pseudoplatanus) polymerizes monolignols. Plant Physiol 99:1162-1168

Wang SY, KS Lewers (2007). Antioxidant capacity and flavonoid content in wild strawberries. J Amer Soc Hortic Sci 132:629-637

Xiao YH, Q Yan, H Ding, M Luo, L Hou, M Zhang, D Yao, HS Liu, X Li, J Zhao, Y Pei (2014). Transcriptome and biochemical analyses revealed a detailed proanthocyanidin biosynthesis pathway in brown cotton fiber. PLoS One 9; Article e86344

$\mathrm{Xu}$ WJ, C Dubos, L Lepiniec (2015). Transcriptional control of flavonoid biosynthesis by MYB-bHLH-WDR complexes. Trends Plant Sci 20:176-185

Xu WJ, L Loïc, D Christian (2014a). New insights toward the transcriptional engineering of proanthocyanidin biosynthesis. Plant Signal Behav 9; Article e28736

Xu WP, H Peng, TB Yang, B Whitaker, LH Huang, JH Sun, P Chen (2014b). Effect of calcium on strawberry fruit flavonoid pathway gene expression and anthocyanin accumulation. Plant Physiol Biochem 82:289-298

Zhang K, K Lu, C Qu, Y Liang, R Wang, Y Chai, JN Li (2013). Gene silencing of BnTT10 Family genes causes retarded pigmentation and lignin reduction in the seed coat of Brassica napus. PLoS One 8; Article e61247

Zhao J, YZ Pang, RA Dixon (2010). The mysteries of proanthocyanidin transport and polymerization. Plant Physiol 153:437-443 